\title{
CONTINUOUS LATTICE ORDERING BY SCHAUDER BASIS CONES ${ }^{1}$
}

\author{
JOHN T. HOFLER
}

\begin{abstract}
Let $(E, \tau)$ be a barrelled Hausdorff space lattice ordered by the cone of an unconditional Schauder basis $\left(x_{n}, f_{n}\right)$. It is shown that under such an ordering $(E, T)$ is a locally convex lattice. Necessary and sufficient conditions are given for the lattice operations to be continuous with respect to the weak topologies on $E$ and its topological dual $E^{\prime}$ : the lattice operations are $\sigma\left(E, E^{\prime}\right)$ continuous on $E$ if and only if $\left\{f_{n}: n \in \omega\right\}$ is a Hamel basis for $E^{\prime}$.
\end{abstract}

1. Preliminaries. Let $(E, \tau)$ be a locally convex space. Let $E^{\prime}$ be the space the all $\tau$-continuous linear functionals on $E$. A $b i$ orthogonal system in $(E, \tau)$ is a sequence $\left\{x_{k}\right\}$ in $E$ and a sequence $\left\{f_{k}\right\}$ in $E^{\prime}$ with the property that $f_{n}\left(x_{m}\right)=\delta_{n, m}$. A Schauder basis for $(E, \tau)$ is a biorthogonal system in $(E, \tau)$ such that for each $x \in E$ we have $x=\sum_{k=1}^{\infty} f_{k}(x) x_{k}$. The series is understood to converge to $x$ in the topology $(E, \tau)$. If $\left(x_{k}, f_{k}\right)$ is a Schauder basis for $(E, \tau)$ and every rearrangement of $\sum_{k=1}^{\infty} f_{k}(x) x_{k}$ converges to $x$, then $\left(x_{k}, f_{k}\right)$ is called an unconditional Schauder basis for $(E, \tau)$.

An ordered vector space is a real vector space $E$ equipped with a transitive, reflexive, antisymmetric relation $\leqq$ satisfying the following conditions:

(a) If $x, y, z \in E$ and $x \leqq y$, then $x+z \leqq y+z$.

(b) If $x, y \in E$ and $\lambda \geqq 0$, then $x \leqq y$ implies $\lambda x \leqq \lambda y$.

The positive cone $K$ in an ordered vector space $E$ is defined by $K=\{x \in E \mid x \geqq \theta\}$, where $\theta$ is the zero vector of $E$. The set $K$ has the properties that $K+K \subseteq K, \lambda K \subseteq K$ for each $\lambda \geqq 0$, and $K \cap$ $-K=\{\theta\}$. A set with the above three properties is called a cone. If $K$ is a cone in a real vector space $E$, then a relation $\leqq$ is defined on $E$ by $x \leqq y$ if $y-x \in K$. With this order $E$ is an ordered vector space with positive cone $K$, i.e. $E$ is ordered by $K$. The terminology in this paper concerning ordered vector spaces is that of [5].

Presented to the Society, November 8, 1968 under the title Schauder basis cones and continuity of the lattice operations in barreled spaces; received by the editors March 27, 1970 and, in revised form, March 1, 1971.

AMS 1969 subject classifications. Primary 4606, 4601; Secondary 0680.

Key words and phrases. Continuous lattice operations, Schauder basis cones, unconditional Schauder basis.

1 This work which was supported in part by NSF Grant GP-7243, is a portion of the Author's Florida State University dissertation written under the direction of Professor C. W. McArthur. 
We assume, unless otherwise stipulated, that $(E, \tau)$ is a barrelled space with unconditional Schauder basis $\left\{x_{n}, f_{n}\right\}$. Furthermore, $E$ is ordered by the cone

$$
K\left\{x_{n}\right\}=\left\{x \in E: f_{n}(x) \geqq 0(n=1,2, \cdots)\right\} .
$$

2. Strong continuity of the lattice operations. We begin with the following theorem.

THEOREM 1. If $K_{\left\{x_{n}\right\}}$ determines a lattice ordering on $E$ (e.g., if $(E, \tau)$ is sequentially complete), then the lattice operations are $\tau$-continuous and hence $(E, \tau)$ is a locally convex lattice.

Proof. By [3, Lemma 7], there exists a generating family $P$ of seminorms for $\tau$ such that $x \in E, \sigma$ a finite subset of positive integers, and $p \in P$ imply $p\left(\sum_{n \in \sigma^{\prime}} f_{n}(x) x_{n}\right) \leqq p\left(\sum_{n \in \sigma} f_{n}(x) x_{n}\right)$ for each $\phi \neq \sigma^{\prime} \subseteq \sigma$. Since $K_{\left\{x_{n}\right\}}$ is normal with respect to $\tau$ [3, Lemma 7], it is sufficient $\left[5\right.$, Proposition 4.5, p. 103] to show the mapping $x \rightarrow x^{+}$is $\tau$-continuous at $\theta$. Suppose $\left\{x_{\alpha}\right\}_{\alpha \in \Omega}$ is a net such that $p\left(x_{\alpha}\right) \stackrel{\alpha}{\rightarrow} 0$ $(p \in P)$. Each $x_{\alpha}$ has a Schauder basis representation of the form $x_{\alpha} \stackrel{\tau}{=} \sum_{n=1}^{\infty} f_{n}\left(x_{\alpha}\right) x_{n}$. It then follows from [3, Lemma 8] that $x_{\alpha}^{+}$ $\stackrel{\tau}{=} \sum_{n=1}^{\infty} \epsilon_{n}^{\alpha} f_{n}\left(x_{\alpha}\right) x_{n}$, where $\epsilon_{n}^{\alpha}=0$ if $f_{n}\left(x_{\alpha}\right)<0$ and $\epsilon_{n}^{\alpha}=1$ if $f_{n}\left(x_{\alpha}\right) \geqq 0$. It then follows from an inequality in McArthur and Retherford [4] that for each positive integer $m$,

$$
\begin{aligned}
p\left(\sum_{n=1}^{m} \operatorname{\epsilon }_{n}^{\alpha} f_{n}\left(x_{\alpha}\right) x_{n}\right) & \leqq 4\left(\sup _{n \in[1, m]}\left|\epsilon_{n}^{\alpha}\right|\right)\left(\sup _{\sigma^{\prime} \subseteq[1, m]} p\left(\sum_{n \in \sigma^{\prime}} f_{n}\left(x_{\alpha}\right) x_{n}\right)\right) \\
& \leqq 4 p\left(\sum_{n=1}^{m} f_{n}\left(x_{\alpha}\right) x_{n}\right) \leqq 4 p\left(x_{\alpha}\right) .
\end{aligned}
$$

Therefore $p\left(x_{\alpha}^{+}\right) \leqq 4 p\left(x_{\alpha}\right) \quad(\alpha \in \Omega)$, and thus the mapping $x \rightarrow x^{+}$is $\tau$-continuous at $\theta$. Since $K_{\left\{x_{n}\right\}}$ is normal with respect to the locally convex topology $\tau$, it follows from [5, Proposition 4.7, p. 104] that $(E, \tau)$ is a locally convex lattice. This completes the proof of the theorem.

Ceitlin [1] established the above result for sequentially complete bornological spaces ordered by the cone of an unconditional Schauder basis. If a locally convex space with unconditional Schauder basis is sequentially complete, then the basis is a bounded-multiplier basis and the associated cone thus defines a lattice ordering on $E$. Saxon cites in his dissertation [6, pp. 78 and 86] an example of a nonsequentially complete barrelled space ordered by the cone of an unconditional Schauder basis. The example is as follows. Let $\left\{b_{i}\right\}=\left\{1 / 2^{2 i}\right\}$ and let $E$ be the subspace of $l_{1}$, spanned by 
$\left(\left\{e_{i}\right\} \cup\left\{\sum_{i=1}^{\infty} b_{i} e_{n_{i}}: n_{i}\right.\right.$ is a subsequence of $\left.\left.\omega\right\}\right)$. Then $E$ is a dense and barrelled subspace of $l_{1}$. The cone associated with $\left\{e_{n}\right\}$, the unit vector basis, defines a lattice ordering on $E$. Thus Theorem 1 generalizes Ceitlin's result.

The following corollary is immediate from Theorem 1 and [5, Proposition 4.17, p. 108].

Corollary 2. If $K_{\left\{x_{n}\right\}}$ defines a lattice ordering on $E$, then $\left(E^{\prime}, \beta\left(E^{\prime}, E\right)\right)$ ordered by $\left.K^{\prime}{ }_{x_{n}}\right\}$ and $\left(E^{\prime \prime}, \beta\left(E^{\prime \prime}, E^{\prime}\right)\right)$ ordered by $\left.K^{\prime \prime}{ }_{\left\{x_{n}\right.}\right\}$ $=\left(K^{\prime}\left(x_{n} \mid\right)^{\prime}\right.$ are locally convex lattices.

Proposition 3. Let $(E, \tau)$ be a locally convex lattice ordered by a cone $K$ such that $\sigma\left(E^{\prime \prime}, E^{\prime}\right)$-bounded subsets of $E^{\prime \prime}$ are $\beta\left(E^{\prime \prime}, E^{\prime}\right)$ bounded, then $\left(E^{\prime}, \beta\left(E^{\prime}, E^{\prime \prime}\right)\right)$ ordered by $K^{\prime}$ is a locally convex lattice.

Proof. Since $\left(E^{\prime \prime}, \beta\left(E^{\prime \prime}, E^{\prime}\right)\right)$, ordered by $K^{\prime \prime}$, is a locally convex lattice, $\beta\left(E^{\prime \prime}, E^{\prime}\right)$ has a neighborhood basis of $\theta$ consisting of solid sets. Thus the class of all solid $\sigma\left(E^{\prime \prime}, E^{\prime}\right)$-bounded subsets is a fundamental system for the class of all $\sigma\left(E^{\prime \prime}, E^{\prime}\right)$-bounded subsets of $E^{\prime \prime}$. Furthermore, by [5, Proposition 4.17, p. 108], $E^{\prime \prime}$ is a lattice ideal in $\left(E^{\prime}\right)^{+}$. Thus the polar of a solid subset of $E^{\prime \prime}$ is a solid subset of $\left(E^{\prime}\right)^{+}$. Thus the polar of a solid subset of $E^{\prime \prime}$ is a solid subset of $E^{\prime}$ by [5, Corollary 4.4, p. 102]. Therefore $\beta\left(E^{\prime}, E^{\prime \prime}\right)$, the topology of uniform convergence on the $\sigma\left(E^{\prime \prime}, E^{\prime}\right)$-bounded subsets of $E^{\prime \prime}$, has a neighborhood basis of $\theta$ consisting of solid convex sets. Thus $\beta\left(E^{\prime}, E^{\prime \prime}\right)$ ordered by $K^{\prime}$ is a locally convex lattice.

Corollary 4. If $K_{\left\{x_{n}\right\}}$ defines a lattice ordering on $E$, then $\left(E^{\prime}, \beta\left(E^{\prime}, E^{\prime \prime}\right)\right)$ ordered by $\left.K^{\prime}{ }_{x_{n}}\right\}$ is a locally convex lattice.

Proof. By Theorem 1 and [5, Corollary 1.10, p. 146], $E^{\prime}$ is complete for $\beta\left(E^{\prime}, E\right)$. Thus every $\sigma\left(E^{\prime \prime}, E^{\prime}\right)$-bounded subset of $E^{\prime \prime}$ is $\beta\left(E^{\prime \prime}, E^{\prime}\right)$-bounded. The conclusion then follows from Proposition 3.

3. Weak continuity of the lattice operations. We now show, with hypotheses similar to the above, that the lattice operations are seldom continuous with respect to the various weak topologies on $E, E^{\prime}$, and $E^{\prime \prime}$. We first prove the following lemma.

Lemma 5. Let $(E, \tau)$ be a locally convex space with Schauder basis $\left(x_{n}, f_{n}\right)$ and $(s)$ denote the space of all real sequences metrized in the usual manner. There exists a linear homeomorphism $\phi$ from $(E, \tau)$ onto a subspace $H$ of $(s)$ such that $\phi\left(x_{i}\right)=e_{i}(i=1,2, \cdots)$ if and only if $\left\{f_{i}:(i=1,2, \cdots)\right\}$ is a Hamel basis for $E^{\prime}$. Here $e_{n}$ is that vector in $(s)$ all of whose coordinates are zero except the nth one. The nth coordinate is 1 . 
Proof. The condition is clearly necessary. To show the condition is sufficient suppose $\left\{f_{i}:(i=1,2, \cdots)\right\}$ is a Hamel basis for $E^{\prime}$ and define a mapping $\phi$ of $E$ into $(s)$ by $\phi(x)=\sum_{i=1}^{\infty} f_{i}(x) e_{i}$ $\left(\sum_{i=1}^{\infty} f_{i}(x) x_{i}=x \in E\right)$. Let $H=\left\{\sum_{i=1}^{\infty} f_{i}(x) e_{i} \in(s): \sum_{i=1}^{\infty} f_{i}(x) x_{i}=x \in E\right\}$. The mapping $\phi$ is clearly linear, 1-1, and on to $H$. The continuity of $\phi$ follows from the definition of $\phi$, the continuity of each $f_{i}$, and the fact that convergence in $(s)$ with respect to the metric is equivalent to coordinatewise convergence. We now show $\phi^{-1}$ is continuous. Let $B \subseteq H$ be bounded. Since $H$ is bornological, it is sufficient to show $\phi^{-1}(B)$ is bounded. Let $f \in E^{\prime}$, then $f=\sum_{i=1}^{n} C_{i} f_{i}$ since $\left\{f_{i}:(i=1,2, \cdots)\right\}$ is a Hamel basis for $E^{\prime}$. Since $g=\sum_{i=1}^{n} C_{i} g_{i}$ belongs to $H^{\prime}$ and $B$ is bounded, it follows that there exists a constant $M_{0} \geqq 0$ such that

$$
\begin{aligned}
M_{0} & \geqq\left|g\left(\phi\left(x_{0}\right)\right)\right|=\left|g\left(\sum_{i=1}^{\infty} f_{i}\left(x_{0}\right) e_{i}\right)\right|=\left|\sum_{i=1}^{\infty} f_{i}\left(x_{0}\right) g\left(e_{i}\right)\right| \\
& =\left|\sum_{i=1}^{n} C_{i} f_{i}\left(x_{0}\right)\right|=\left|f\left(x_{0}\right)\right| \quad\left(x_{0}=\sum_{i=1}^{\infty} f_{i}\left(x_{0}\right) x_{i} \in \phi^{-1}(B)\right) .
\end{aligned}
$$

Hence $\phi^{-1}(B)$ is bounded, and thus $\phi^{-1}$ is continuous.

THEOREM 6. If $K_{\left\{x_{n}\right\}}$ defines a lattice ordering then the following are equivalent.

(i) The lattice operations are $\sigma\left(E, E^{\prime}\right)$-continuous.

(ii) $\left\{f_{i} \mid(i=1,2, \cdots)\right\}$ is a Hamel basis for $E^{\prime}$.

(iii) There exists a linear homeomorphism $\phi$ from $(E, \tau)$ onto a barrelled subspace of $(s)$ such that $\phi\left(x_{i}\right)=e_{i}$.

(iv) $\left(x_{i}, f_{i}\right)$ is a weakly equicontinuous Schauder basis for $E$ (i.e., $\left\{S_{n} \in L(E, E): S_{n}(x)=\sum_{i=1}^{n} f_{i}(x) x_{i}(x \in E)\right\}$ is a weakly equicontinuous family of projections on $E$ ).

Proof. T. Cook [2] showed the equivalence of (ii) and (iv). The equivalence of (ii) and (iii) follows immediately from the preceding lemma.

To show (i) implies (ii) suppose the lattice operations are $\sigma\left(E, E^{\prime}\right)$ continuous but $\left\{f_{i} \mid(i=1,2, \cdots)\right\}$ is not a Hamel basis for $E^{\prime}$. Since $\left(f_{i}, x_{i}\right)$ is a $\sigma\left(E^{\prime}, E\right)$ unconditional Schauder basis for $E^{\prime}$ but not a Hamel basis, there exists $\sum_{i=1}^{\infty} f\left(x_{i}\right) f_{i}=f \in E^{\prime}\left(\sigma\left(E^{\prime}, E\right)\right)$ and a subsequence $\left\{n_{k}\right\}_{k=1}^{\infty}$ of the positive integers such that $f\left(x_{n_{k}}\right) \neq 0$ $(k=1,2, \cdots)$. Since $E$ is barrelled and hence $E^{\prime}$ is $\sigma\left(E^{\prime}, E\right)$ sequentially complete, we may assume each $f\left(x_{i}\right) \geqq 0(i=1,2, \cdots)$. Let $f^{k}=\sum_{i=1}^{n_{k}} f\left(x_{i}\right) f_{i}$. Then 
$f^{k} \in[0, f]=\left\{g \in E^{\prime} \mid 0 \leqq g(x) \leqq f(x)(x \in K)\right\} \quad(k=1,2, \cdots)$,

and $\left\{f^{k} \mid k=1,2, \cdots\right\}$ is a linearly independent set. But $[0, f]$ must be contained in a finite dimensional subspace of $E^{\prime}$ by [5, Proposition 2.13 , p. 134]. Hence $\left\{f_{i} \mid(i=1,2, \cdots)\right\}$ must be a Hamel basis for $E^{\prime}$.

To show (ii) implies (i) it is sufficient to show the mapping $x \rightarrow x^{+}$ is $\sigma\left(E, E^{\prime}\right)$-continuous at $\theta$ since $K_{\left\{x_{n}\right\}}$ is $\sigma\left(E, E^{\prime}\right)$-normal. Suppose $\left\{x_{\alpha}\right\}_{\alpha \in \Omega}$ is a net in $E$ such that $\left\{x_{\alpha}\right\}_{\alpha \in \Omega}$ weakly converges to $\theta$. As in the proof of Theorem 1 each $x_{\alpha} \stackrel{\tau}{=} \sum_{i=1}^{\infty} f_{i}\left(x_{\alpha}\right) x_{i}$ and $x_{\alpha}^{+} \stackrel{\tau}{=} \sum_{i=1}^{\infty} \epsilon_{i}^{\alpha} f_{i}\left(x_{\alpha}\right) x_{i}$, where $\epsilon_{i}^{\alpha}=0$ if $f_{i}\left(x_{\alpha}\right)<0$ and $\epsilon_{i}^{\alpha}=1$ if $f_{i}\left(x_{\alpha}\right) \geqq 0$. It then follows from (ii) in a straightforward manner that $f\left(x_{\alpha}^{+}\right) \underset{\alpha}{\rightarrow} 0$ for every $f \in E^{\prime}$.

The sequence spaces $l^{P}(P \geqq 1)$ and $c_{0}$ considered as subspaces of $(s)$ with the relative topology have unconditional Schauder bases which define a lattice ordering and satisfy the hypotheses of Lemma 5. However, there appears to be a paucity of barrelled subspaces of (s) which satisfy the hypotheses of Lemma 5 and Theorem 6. In fact, for sequentially complete barrelled spaces we have the following corollary.

Corollary 7. If $(E, \tau)$ is sequentially complete and the lattice operations are $\sigma\left(E, E^{\prime}\right)$-continuous, then $E$ is linearly homeomorphic to the space $(s)$.

Suppose $(E, \tau)$ is a locally convex space ordered by a cone $K$ and $E^{\prime}$ is the topological dual of $E$ ordered by $K^{\prime}=\{f \in E: f(x) \geqq 0(x \in K)\}$. If $K_{\left\{x_{n}\right\}}$ is the cone associated with a Schauder basis, then $K^{\prime}\left\{x_{n}\right\}$ $=\left\{f \in E^{\prime} \mid f\left(x_{n}\right) \geqq 0(n=1,2, \cdots)\right\}$ and its dual taken in $E$ is $K_{\left\{x_{n}\right\}}$. This observation and the methods used in establishing the equivalence of (i) and (ii) in Theorem 6 can be used to prove the following corollary.

Corollary 8. Let $(E, \tau)$ be sequentially complete. If $K_{\left\{x_{n}\right\}}$ defines a lattice ordering on $E$ then the lattice operations in $E^{\prime}$ ordered by $K^{\prime}\left\{x_{n}\right\}$ are $\sigma\left(E^{\prime}, E\right)$-continuous if and only if $\left\{x_{i} \mid(i=1,2, \cdots)\right\}$ is a Hamel basis for $E$.

Corollary 8 indicates that under the hypotheses given, the lattice operations are seldom $\sigma\left(E, E^{\prime}\right)$-continuous. In fact a simple category argument shows that for no Frechet space can a Schauder basis also be a Hamel basis. The topological dual $E^{\infty}$ of $(s)$ satisfies the given condition, and any other space satisfying this condition must certainly be algebraically isomorphic to the vector space $E^{\infty}$ of finitely 
nonzero real sequences. If one only requires weak sequential continuity of the lattice operations then much more is true than has been stated in the above results. For example, in any nuclear Frechet lattice the lattice operations are weakly sequentially continuous. ${ }^{2}$

\section{REFERENCES}

1. Ja. M. Cě̆tlin, Unconditional basis and semiorderedness, Izv. Vysš. Ucebn. Zaved. Matematika 1966, no. 2 (51), 98-104; English transl., Amer. Math. Soc. Transl. (2) 90 (1969), 17-25. MR 33 \#6362.

2. T. A. Cook, Weakly equicontinuous Schauder bases, Proc. Amer. Math. Soc. 23 (1969), 536-537. MR $40 \# 1748$.

3. C. W. McArthur, Convergence of monotone nets in ordered topological vector spaces, Studia Math. 34 (1970), 1-16.

4. C. W. McArthur and J. R. Retherford, Some applications of an inequality in locally convex spaces, Trans. Amer. Math. Soc. 137 (1969), 115-123. MR 39 \#748.

5. A. L. Peressini, Ordered topological vector spaces, Harper and Row, New York, 1967. MR $37 \# 3315$.

6. S. A. Saxon, Basis cone base theory, Dissertation, Florida State University, Tallahassee, Fla., 1969.

University of South Carolina, Columbia, South Carolina 29208

2 This fact was pointed out by the referee. 